\title{
Interação genótipo-ambiente sobre os pesos aos 205 e 365 dias de idade em bovinos da raça Nelore em diferentes regiões do Brasil
}

\author{
Genotype-environment interaction on the weights at 205 and 365 days of age in Nellore \\ cattle in different regions of Brazil
}
CARVALHO, Caio Victor Damasceno ${ }^{1 *}$; BITTENCOURT, Thereza Cristina Calmon ${ }^{2}$; LÔBO, Raysildo Barbosa ${ }^{3}$; PINTO, Luiz Fernando Batista²; NASCIMENTO, Misael Caldas $^{4}$

\footnotetext{
${ }^{1}$ Universidade Federal da Bahia, Escola de Medicina Veterinária e Zootecnia, Programa de PósGraduação em Zootecnia, Salvador, Bahia, Brasil,

${ }^{2}$ Universidade Federal da Bahia, Escola de Medicina Veterinária e Zootecnia, Departamento de Produção Animal, Salvador, Bahia, Brasil.

${ }^{3}$ Associação Nacional de Criadores e Pesquisadores, Ribeirão Preto, São Paulo, Brasil.

${ }^{4}$ Universidade Federal da Bahia, Escola de Medicina Veterinária e Zootecnia, Programa de PósGraduação em Ciência Animal nos Trópicos, Salvador, Bahia, Brasil.

*Endereço para correspondência: caiovictor3@gmail.com
}

\section{RESUMO}

Dados de pesos à desmama ajustados para 205 dias (P205) e ao ano ajustados para 365 dias (P365), de animais da raça Nelore provenientes de 29 fazendas de diferentes estados brasileiros distribuídos entre as regiões Norte, Sudeste e Centro-Oeste, participantes do Programa de melhoramento genético da raça Nelore (Nelore Brasil), foram utilizados para avaliar a presença da interação genótipo-ambiente (IGA), bem como estimar as herdabilidades direta (P365 e P205) e materna (P205) pelo método de máxima verossimilhança restrita com auxílio do programa MTDFREML. O modelo estatístico utilizado incluiu efeitos fixos de grupo contemporâneo, idade da vaca ao parto (covariável) e efeitos aleatórios genéticos direto (P205 e P365) e materno (P205). As correlações genéticas estimadas para as características P205 e P365 em cada uma das regiões, Norte (R1), Sudeste (R2) e Centro-Oeste (R3), foram, para P205: 0,85 $\pm 0,11$ entre R1 e R2; 0,96 $\pm 0,07$ entre R1 e R3; e, 0,95 $\pm 0,07$ entre R2 e R3, e para P365: 0,72 $\pm 0,09$ entre R1 e R2; 0,83 $\pm 0,07$ entre R1 e R3; e $0,85 \pm 0,08$ entre $\mathrm{R} 2$ e R3. Esses resultados indicam que, na desmama (P205), o efeito da IGA não foi observado, porém para o peso ao ano (P365), na combinação envolvendo as regiões Norte/Sudeste foi verificado efeito da IGA.

Palavras-chave: bovinos de corte, peso à desmama, peso ao ano.

\section{SUMMARY}

Data of weaning weight (205BW) and at year (365BW) of Nellore cattle from 29 farms in several Brazilian states of three distinct regions: North, Southeast and Midwest, all participants of the program of Nellore breeding (Nellore Brazil), were used to assess the presence of genotype by environment interaction (GEI) as well as estimate direct heritability (205BW and $365 \mathrm{BW}$ ) and maternal (205BW) by restricted maximum likelihood method with the aid of MTDFREML. The statistical model used included fixed effects of contemporary group, age of dam (covariate) and random direct genetic effects (205BW and 365BW) and maternal (205BW). The estimated genetic correlation for $205 \mathrm{BW}$ and $365 \mathrm{BW}$ in each of the regions, North (R1), Southeast (R2) and Midwest (R3), were respectively: $0.85 \pm 0.11$, $0.96 \pm 0.07$ and $0.95 \pm 0.07$ for R1/R2, R1/R3 and $\mathrm{R} 2 / \mathrm{R} 3$, the evaluation of weaning weight, $0.72 \pm 0.09,0.83 \pm 0.07$ and $0.85 \pm 0.08$, for R1/R2, $\mathrm{R} 1 / \mathrm{R} 3$ and R2/R3, in the evaluation year. These results indicate that, at weaning $(205 \mathrm{BW})$, the effect of GEI was not observed, but in the weight at year (P365), the combination involving North/Southeast effect was verified of the GEI.

Keywords: beef cattle, weight the year, weaning weight. 


\section{INTRODUÇÃO}

O maior poder aquisitivo da população brasileira na última década, assim como o crescimento da população mundial, levaram ao aumento da demanda mundial por alimentos. Neste cenário, $\mathrm{o}$ Brasil se destaca pela capacidade de aumentar a sua produção agropecuária, principalmente a de proteína de origem animal, onde sempre se sobressaiu internacionalmente.

Com o rebanho bovino de 209,5 milhões de animais em 2010 (IBGE, 2011), e dimensões continentais, o Brasil possui regiões com marcadas diferenças climáticas, promovendo mudanças em importantes fatores ligados à produção animal, como clima, solo, alimentação e manejo (TEIXEIRA et al., 2006).

O valor observado de uma característica, o fenótipo, é dependente do potencial genético do indivíduo e do ambiente no qual é produzido. Esta é uma simplificação do que ocorre biologicamente, o que significa dizer que a genética e o ambiente influenciam de forma aditiva e independente no fenótipo (FRIDRICH et al., 2005; FARIA et al., 2011).

Entretanto, a mudança de desempenho do mesmo genótipo em ambientes diferentes é devido à interação genótipo-ambiente (IGA), ou seja, quando um genótipo é melhor em determinado ambiente e não em outro (CORRÊA et al., 2009).

A IGA pode também provocar alterações nas variâncias genéticas, fenotípicas e ambientais e resultar em mudanças nas estimativas dos parâmetros genéticos e fenotípicos, implicando na possibilidade de mudanças nos critérios de seleção, dependendo do ambiente (ALENCAR et al., 2005).

Trabalhos publicados avaliando a influência da IGA em bovinos de corte no Brasil, concluíram que animais identificados como melhoradores em determinado rebanho podem não apresentar os melhores fenótipos se transferidos para condições ambientais diferentes (FRIDRICH et al., 2008; SOUZA et al., 2008).

Uma consideração a ser feita refere-se à escolha do ambiente onde se deve praticar a seleção dos animais de reprodução, porque progênies de um mesmo reprodutor podem não repetir $o$ desempenho dos pais, caso sejam criadas em regiões diferentes, evidenciando a necessidade de cuidados na compra de reprodutores e, ou sêmen, em razão da existência de IGA (LOPES et al., 2008).

Desta forma, objetivo-se avaliar a presença da IGA e estimar os parâmetros genéticos do peso ao desmame (P205) e ao ano (P365), de bovinos da raça Nelore criados em três regiões do Brasil (Centro-Oeste, Norte e Sudeste).

\section{MATERIAL E MÉTODOS}

Foram utilizados dados coletados pela ANCP (Associação Nacional de Criadores e Pesquisadores) oriundos de 29 rebanhos brasileiros, participantes do Programa de Melhoramento Genético da Raça Nelore (Nelore Brasil). O banco de dados original é constituído por pesos ajustados em diferentes idades, e os arquivos formados para as análises continham informações cadastrais de 38486 animais para o peso ajustado aos 205 dias e 58095 para o peso ajustado aos 365 dias de idade, todos de bovinos da raça Nelore, nascidos entre 2001 e 2009.

As características estudadas foram os pesos corporais dos animais aos 205 (P205) e aos 365 (P365) dias de idade padronizados por interpolação, e o 
intervalo considerado entre as pesagens foi, no máximo, de 90 dias, de acordo com o sistema de avaliação genética adotado pela ANCP.

Para cada uma das características foram formados dois arquivos para as análises. O primeiro continha os dados do animal, pai, mãe, grupo contemporâneo, idade da vaca ao parto e os pesos padronizados para as idades em estudo, considerados como características distintas em cada região. $\mathrm{O}$ segundo arquivo continha informações do pedigree, formado pelos dados recodificados do animal, do pai e da mãe. Os arquivos para avaliar o efeito da interação genótipo-ambiente sobre as características analisadas nas diversas regiões brasileiras, foram constituídos apenas com informações de animais criados exclusivamente a pasto e com suplementação mineral. Os estados que possuíam informações contidas no banco de dados foram agrupados em três regiões distintas: Norte, Sudeste, e Centro-Oeste.

Para a formação dos grupos de contemporâneos os animais foram agrupados quanto ao sexo, fazenda, ano e estação de nascimento. Foram consideradas quatro estações de nascimento: outubro a dezembro, janeiro a março, abril a junho e julho a setembro.

A conectividade entre as regiões foi definida por "touros de conexão", isto é, os touros deveriam ter número mínimo de cinco progênies em cada uma das região. Para avaliar a presença da IGA, as características foram avaliadas em análises bicaracterística entre as seguintes combinações: Norte x Sudeste; Norte x Centro-Oeste e Sudeste x Centro-Oeste.

As análises estatísticas dos pesos ajustados às idades-padrão foram feitas com o auxílio do procedimento Generalized Linear Models (GLM), do pacote estatístico Statistical Analyses Systems (SAS Institute, 1997), com o objetivo de avaliar a importância dos efeitos de ambiente sobre os pesos estudados. Na Tabela 1 encontram-se algumas estatísticas descritivas das características das amostras estudadas, após a eliminação dos dados como referidos anteriormente.

Tabela 1. Estatística descritiva do arquivo com informações do peso aos 205 e aos 365dias de idade nas três regiões estudadas

\begin{tabular}{|c|c|c|c|}
\hline Item & Norte & Sudeste & Centro - Oeste \\
\hline \multicolumn{4}{|c|}{ Peso aos 205 dias de idade - P205 } \\
\hline Número de animais & 8085 & 9337 & 20923 \\
\hline Número de animais na $A^{-1}$ & 12230 & 14896 & 35148 \\
\hline Média \pm DP & $187,34 \pm 14,91$ & $196,51 \pm 18,32$ & $183,53 \pm 16,64$ \\
\hline Coeficiente de variação & $7,96 \%$ & $9,32 \%$ & $9,08 \%$ \\
\hline \multicolumn{4}{|c|}{ Peso aos 365 dias de idade - P365 } \\
\hline Número de animais & 9263 & 12328 & 36504 \\
\hline Número de animais na $A^{-1}$ & 13807 & 18700 & 57373 \\
\hline Média \pm DP & $237,8 \pm 23,9$ & $254,8 \pm 27,2$ & $232,1 \pm 25,2$ \\
\hline Coeficiente de variação & $10,05 \%$ & $10,68 \%$ & $10,75 \%$ \\
\hline
\end{tabular}

$\mathrm{A}^{-1}=$ matriz de parentesco; $\mathrm{DP}=$ desvio padrão. 
Para estimação dos componentes de (co)variância e predição dos valores genéticos, utilizou-se o programa Multiple Trait Derivative-free Restricted Maximum Likelihood (MTDFREML), descrito por Boldman et al. (1995), que aplica o método de máxima verossimilhança restrita, com algoritmos livres de derivadas, sob modelo animal, com inclusão da matriz de parentesco.

Os critérios de convergência adotados pelo programa foram: a) valor da predição de variância do simplex para localizar o mínimo de -2 Log L (em que $\mathrm{L}=$ função de máxima verossimilhança) $10^{-6}$ em modelos com múltiplos parâmetros; e b) não haver alteração no valor das estimativas do simplex $-2 \log$ de verossimilhança $10^{-9}$ em quatro repetições sucessivas. A cada convergência, o programa foi reiniciado, usando os valores obtidos na análise anterior como valores iniciais por quatro repetições sucessivas. Os valores genéticos foram estimados a partir dos componentes de variância.

Para fins de comparação e melhor avaliação dos resultados e obtenção dos parâmetros genéticos, foram feitas análises unicaracterísticas, com $\mathrm{o}$ emprego do modelo estatístico que considerou o efeito fixo de grupo de contemporâneo, a idade da vaca ao parto como covariável (efeito linear e quadrático) para P205 e P365, sendo avaliados os efeitos aleatórios genético aditivo direto para P205 e P365, e efeito genético aditivo materno utilizados apenas para o P205.

Os efeitos da IGA foram obtidos pelas correlações genéticas entre os pesos, considerando a mesma característica (P205 e P365) como característica diferente nas regiões avaliadas (análise bicaracterística).

Os dados foram analisados de duas formas: uma característica por vez (análise unicaracterística) ou duas características (análise bicaracterística), onde os pesos foram considerados como características distintas em cada uma das regiões.

$\mathrm{O}$ modelo linear para descrever as características foi definido como:

$$
\mathbf{Y}=\mathbf{x} \boldsymbol{\beta}+\mathbf{z}_{1 \mathrm{u}}+\mathbf{z}_{2 \mathrm{~m}}+\mathbf{z}_{3 \mathrm{p}}+\mathbf{e},
$$

Em que:

$\mathbf{Y}=$ vetor das observações,

$\mathbf{X}=$ matriz de incidência dos efeitos fixos,

$\beta=$ vetor dos efeitos fixos,

$\mathbf{Z}_{1}=$ matriz de incidência do efeito genético direto de cada animal,

$\mathbf{u}=$ vetor de efeitos genéticos diretos aleatórios,

$\mathbf{Z}_{\mathbf{2}}=$ matriz de incidência dos valores genéticos maternais,

$\mathbf{m}=$ vetor de efeitos genéticos maternais aleatórios,

$\mathbf{Z}_{\mathbf{3}}=$ matriz de incidência do efeito do ambiente permanente,

$\mathbf{p}=$ vetor de efeitos aleatórios do ambiente permanente

$\mathbf{e}=$ vetor de efeitos residuais aleatórios.

Para a característica P365 o modelo utilizado apenas não continha o termo $\mathrm{Z}_{2} \mathrm{~m}$, correspondente aos efeitos aditivos maternos.

Nas análises unicaracterística com o modelo completo, as distribuições e matriz de (co)variâncias dos efeitos aleatórios pode ser definida como:

$$
\begin{aligned}
& E[\boldsymbol{\alpha}]=E[m]=E[p]=E[e]=0 \\
& E\left[y \mid \alpha_{z} m, p\right]=X \beta+Z_{1 a}+Z_{2 m}+Z_{3 p}
\end{aligned}
$$

Para as análises conjuntas (bicaráter) a distribuição e matriz de (co)variâncias poderiam ser definidas como a seguir, onde os subscritos 1 e 2 referem-se às duas características analisadas.

$\mathrm{E}\left[\begin{array}{l}y_{1} \\ y^{\mathbf{z}}\end{array}\right]=\left[\begin{array}{l}X_{1} \beta \\ X_{2} \beta\end{array}\right]$ 
$E[\boldsymbol{\alpha}]=E[m]=E[p]=E[e]=0$,

para ambas características,

$E\left[y_{1} \mid a_{1}, m_{1}, p_{1}\right]=X_{1} \beta_{1}+Z_{11 a 1}+Z_{21 m 1}+Z_{31 p 1}$ para a característica 1

$E\left[y_{2} \mid a_{2}, m_{2}, p_{2}\right]=X_{2} \beta_{2}+Z_{12 \alpha 2}+Z_{22 m 2}+Z_{32 p 2}$,

para a característica 2 .

\section{RESULTADOS E DISCUSSÕES}

Os valores das correlações entre os efeitos direto e materno para P205 dias de idade foram, respectivamente, iguais a: $-0.43,0,71$ e $-0,36$ (Tabela 2), para as regiões Norte, Sudeste e Centro-Oeste. As correlações negativas observadas nas regiões Norte e Centro-Oeste, evidenciam um antagonismo entre os efeitos direto e materno. Nesse caso, a seleção para incrementar o efeito materno poderia causar redução nos pesos ao desmame dos animais. Diferente do observado na região Sudeste, onde a correlação foi positiva, indicando que a seleção para o efeito materno poderia levar a um aumento no peso ao desmame dos animais.

Eler et al. (2000), Sarmento et al. (2003) e Faria et al. (2011) também encontraram correlações negativas para P205 (-0,24, 0,77 e $-0,43$, respectivamente), entre os efeitos direto e materno ao trabalharem com zebuínos. Ribeiro et al. (2009) encontraram para o P205 correlações entre os efeitos direto e materno variando de $-0,09$ a 0,28 . Os resultados encontrados também estão de acordo com os de Fridrich et al. (2008) para a mesma característica em bovinos Nelore na regiões Norte e Centro-Oeste $(-0,39$ e -0,49 respectivamente), diferindo apenas do resultado encontrado para a região Sudeste onde o valor apresentado pelo autor foi de $-0,27$.

Tabela 2. Variâncias genética aditiva direta $\left(\sigma_{a}^{2}\right)$, genética aditiva materna $\left(\sigma^{2} m\right)$, fenotípica $\left(\sigma_{p}^{\mathbf{z}}\right)$, residual $\left(\sigma_{\theta}^{\mathbf{z}}\right)$, covariância entre os efeitos genético direto e materno $\left(\sigma_{a m}\right)$, herdabilidades direta $\left(\boldsymbol{h}_{a}^{2} \mathrm{a}\right)$ e materna $\left(\boldsymbol{h}_{\mathfrak{m}}^{2}\right)$ e correlação genética entre os efeitos direto e materno $\left(\boldsymbol{R r} g_{a m}\right)$ obtidas em análise unicaracterística do peso aos 205 (P205) dias de idade, em cada uma das regiões Norte (R1), Sudeste (R2) e Centro-Oeste (R3)

\begin{tabular}{lcccccccc}
\hline Região & $\sigma_{a}^{2}$ & $\sigma_{m}^{2}$ & $\sigma_{y}^{2}$ & $\sigma_{a}^{2}$ & $\sigma_{\pi m}$ & $h_{a x}^{2}$ & $h_{m}^{2}$ & $(\boldsymbol{R} g)_{\operatorname{sim}}$ \\
\hline R1 & 9,96 & 31,49 & 171,92 & 145,70 & $-7,62$ & $0,06 \pm 0,01$ & $0,18 \pm 0,02$ & $-0,43 \pm 0,19$ \\
R2 & 12,02 & 17,20 & 232,09 & 182,48 & 10,20 & $0,05 \pm 0,01$ & $0,07 \pm 0,03$ & $0,71 \pm 0,72$ \\
R3 & 13,69 & 30,65 & 131,82 & 102,30 & $-7,41$ & $0,10 \pm 0,03$ & $0,23 \pm 0,03$ & $-0,36 \pm 0,25$ \\
\hline
\end{tabular}

As estimativas de herdabilidade também variaram entre regiões. Os valores obtidos para herdabilidade direta e materna foram 0,06 e 0,18 (Norte), 0,05 e 0,07 (Sudeste), 0,1 e 0,23 (CentroOeste). Fridrich et al. (2008) estimaram herdabilidade direta e materna para peso aos 205 dias de idade para a raça Nelore entre as mesmas regiões estudadas e encontraram valores de 0,21 e 0,29 (Norte), 0,16 e 0,17 (Sudeste), 0,29 e 0,27 (Centro-Oeste). Yokoo et al. (2007) e Souza et al. (2008) estudando animais da raça Nelore estimaram herdabilidade direta variando de 0,27 a 0,34 . Observa-se que nas regiões avaliadas os valores de herdabilidade 
foram mais baixos do que os valores médios observados na literatura.

Conforme revisão elaborada por Lôbo et al. (2000), utilizando 59 referências de bovinos de corte criados nos trópicos, a herdabilidade direta média para o P205 é de 0,30. As estimativas de herdabilidade materna foram semelhantes às encontradas por Alencar et al. (2005) e Fridrich et al. (2005), para as raças Canchim e Tabapuã, respectivamente.
Nota-se que as estimativas de herdabilidade direta foram menores do que as herdabilidades maternas, ou seja, indicando influências do efeito materno sobre o peso ao desmame, já que até o desmama os bezerros possuem grande dependência das vacas.

Para o peso ao ano (P365) também foram realizadas análises uni $\mathrm{e}$ bicaracterística, no entanto, só foram avaliados os efeitos genéticos aditivos diretos (Tabela 3).

Tabela 3. Variâncias genética aditiva ( $\left.\sigma_{a}^{\mathbf{z}}\right)$, fenotípica ( $\left.\sigma_{p}^{\mathbf{z}}\right)$, residual ( $\left.\sigma_{e}^{\mathbf{z}}\right)$ e herdabilidade direta $\left(\boldsymbol{h}_{\mathbb{a}}^{\mathbf{2}}\right)$ obtidas em análise unicaracterística do peso aos $365(\mathrm{P} 365)$ dias de idade em bovinos Nelore, nas regiões Norte, Sudeste e Centro-Oeste

\begin{tabular}{lrccc}
\hline Região & \multicolumn{1}{c}{$\boldsymbol{\sigma}_{a}^{2}$} & $\sigma_{p}^{2}$ & $\sigma_{e}^{2}$ & $\boldsymbol{h}_{a}^{2}$ \\
\hline Norte & 91,40 & 283,25 & 191,85 & $0,32 \pm 0,02$ \\
Sudeste & 116,82 & 403,12 & 286,30 & $0,29 \pm 0,03$ \\
Centro-Oeste & 80,42 & 281,66 & 201,24 & $0,29 \pm 0,04$ \\
\hline
\end{tabular}

Os valores encontrados para a herdabilidade direta para P365 variaram de 0,29 a 0,32 entre as três regiões estudadas, estes valores são considerados de média magnitude, assim como os valores médios encontrados na literatura para bovinos Nelore, com variação de 0,27 a 0,48 (BITTENCOURT et al., 2002; YOKOO et al., 2007; BOLIGON et al., 2008). Fridrich et al. (2005) obtiveram herdabilidade direta de $0,20 \mathrm{e}$ 0,50 para as regiões Sudeste e CentroOeste respectivamente, trabalhando com animais da raça Tabapuã.

Os valores de herdabilidade encontrados estão de acordo com os encontrados por Fridrich et al. (2008) em bovinos Nelore, 0,13, 0,29 e 0,20, para as regiões Sudeste, Centro-Oeste e Norte, respectivamente.

O menor valor para o componente de variância residual estimado $(191,85)$ foi para a região Norte, o que sugere condições ambientais dentro desta região mais equilibradas que nas outras regiões avaliadas.

São várias as razões para explicar a variação dos componentes de (co)variância e as estimativas de herdabilidade de uma região para outra. Segundo Toral et al. (2004) a herdabilidade é uma propriedade da população e do ambiente no qual o animal vive, enquanto a variância ambiental é dependente das condições de manejo, clima e sanidade. Uma maior variação ambiental reduz a herdabilidade, enquanto uma maior uniformidade provoca aumento da herdabilidade.

As estimativas das variâncias e covariâncias genéticas aditivas direta, para P205 e P365 e materna para P205, fenotípicas e residuais, considerando cada uma destas características como distintas nas diferentes regiões, são apresentadas nas Tabelas 4, 5 e 6 . 
As correlações genéticas encontradas, considerando P205 nas diferentes regiões como características distintas, foram: $0,85 \pm 0,11,0,96 \pm 0,07$ e $0,95 \pm 0,07$ para R1/R2, R1/R3 e R2/R3, respectivamente (Tabela 7).

Os resultados das correlações envolvendo as regiões Sudeste/Centro-Oeste estão de acordo com os apresentados por Fridrich et al. (2005) para bovinos Tabapuã, e com Ribeiro et al. (2009) também para a raça Nelore. Para as combinações envolvendo as regiões Norte/Sudeste e Norte/Centro-Oeste a IGA também não foi observada por Fridrich et al. (2008) trabalhando com animais da raça Nelore.

Os valores das correlações genéticas encontradas para as diferentes regiões foram altas, próximas a 1,0, sugerindo que os mesmos grupos gênicos são responsáveis pela expressão das duas características. Desta forma não houve influência da interação genótipoambiente sobre o P205 nas três regiões estudadas

Para o P365 as correlações genéticas $(R g)$ encontradas indicam a presença da IGA entre as regiões Norte/Sudeste, $R g=0,72 \pm 0,09$ (Tabela 8).

Estes valores, no entanto, vão de encontro ao obtido por Araújo et al. (2011) para animais Nelore, avaliados em diferentes estados distribuídos entre as regiões Sudeste e Centro-Oeste, indicando presença da IGA com $R g$ igual a 0,71 . Assim como os resultados apresentados por Fridrich et al. (2008) para bovinos da raça Nelore distribuídos entre as mesmas regiões estudadas, Norte/Sudeste a $R g$ igual a 0,83 , onde não foi observado o efeito da IGA.

Tabela 4. Estimativas de variâncias genéticas aditiva direta $\left(a_{1}-a_{2}\right)$ e materna $\left(m_{1}-m_{2}\right)$ (diagonal) e covariâncias (fora da diagonal), obtidas pela análise conjunta do peso aos 205 (P205) dias de idade em bovinos Nelore, consideradas como características distintas nas combinações das regiões Norte, Sudeste e CentroOeste

\begin{tabular}{|c|c|c|c|c|}
\hline \multicolumn{5}{|c|}{ Norte (1) x Sudeste (2) } \\
\hline & a1 & a2 & $\mathrm{ml}$ & $\mathrm{m} 2$ \\
\hline a1 & 10,57 & - & - & - \\
\hline a2 & 10,50 & 14,38 & - & - \\
\hline $\mathrm{m} 1$ & $-8,01$ & $-3,36$ & 31,79 & - \\
\hline $\mathrm{m} 2$ & 0,74 & 3,37 & 16,18 & 23,90 \\
\hline \multicolumn{5}{|c|}{ Norte (1) x Centro-Oeste (3) } \\
\hline & a1 & $\mathrm{a} 2$ & $\mathrm{ml}$ & $\mathrm{m} 2$ \\
\hline a1 & 10,91 & - & - & - \\
\hline a2 & 12,23 & 14,82 & - & - \\
\hline $\mathrm{ml}$ & $-6,98$ & $-7,111$ & 30,57 & - \\
\hline $\mathrm{m} 2$ & $-5,71$ & $-11,15$ & $-17,32$ & 34,53 \\
\hline \multicolumn{5}{|c|}{ Sudeste (2) x Centro-Oeste (3) } \\
\hline & al & a2 & $\mathrm{ml}$ & $\mathrm{m} 2$ \\
\hline al & 14,03 & & - & - \\
\hline a2 & 13,37 & 13,88 & - & - \\
\hline $\mathrm{ml}$ & 7,75 & 3,24 & 19,46 & - \\
\hline $\mathrm{m} 2$ & $-2,37$ & $-8,17$ & 20,35 & 31,30 \\
\hline
\end{tabular}


Tabela 5. Estimativas das variâncias fenotípica $\left(\mathrm{p}_{1}-\mathrm{p}_{2}\right)$ e residual $\left(\mathrm{e}_{1}-\mathrm{e}_{2}\right)$ (diagonal) e covariâncias (fora da diagonal), obtidas pela análise conjunta do peso aos 205 (P205) dias de idade em bovinos Nelore, consideradas como características distintas nas combinações das regiões Norte, Sudeste e Centro-Oeste

\begin{tabular}{|c|c|c|c|c|}
\hline \multicolumn{5}{|c|}{ Norte (1) x Sudeste (2) } \\
\hline & p1 & p2 & e1 & e2 \\
\hline p1 & 171,65 & & & \\
\hline p2 & 24,06 & 225,62 & & \\
\hline e1 & - & - & 145,31 & \\
\hline e2 & - & - & - & 180,06 \\
\hline \multicolumn{5}{|c|}{ Norte (1) x Centro-Oeste (3) } \\
\hline & $\mathrm{p} 1$ & $\mathrm{p} 2$ & e1 & $\mathrm{e} 2$ \\
\hline p1 & 172,79 & & & \\
\hline p2 & 16,73 & 128,42 & & \\
\hline e1 & - & - & 145,26 & \\
\hline $\mathrm{e} 2$ & - & - & - & 101,37 \\
\hline \multicolumn{5}{|c|}{ Sudeste (2) x Centro-Oeste (3) } \\
\hline & p1 & p2 & el & e2 \\
\hline p1 & 229,99 & & & \\
\hline p2 & 34,60 & 131,11 & & \\
\hline e1 & - & - & 180,99 & \\
\hline e2 & - & - & - & 102,27 \\
\hline
\end{tabular}

Tabela 6. Estimativas das variâncias fenotípica $\left(\mathrm{p}_{1}-\mathrm{p}_{2}\right)$ e residual $\left(\mathrm{e}_{1}-\mathrm{e}_{2}\right)$ (diagonal) e covariâncias (fora da diagonal), obtidas pela análise conjunta do peso aos 365 (P365) dias de idade em bovinos Nelore, consideradas como características distintas nas combinações das regiões Norte, Sudeste e Centro-Oeste

\begin{tabular}{|c|c|c|c|c|}
\hline \multicolumn{5}{|c|}{ Norte (1) x Sudeste (2) } \\
\hline & p1 & p2 & e1 & e2 \\
\hline p1 & 281,82 & & - & - \\
\hline p2 & 71,84 & 404,74 & - & - \\
\hline e1 & - & - & 201,00 & - \\
\hline e2 & - & - & - & 282,60 \\
\hline \multicolumn{5}{|c|}{ Norte (1) x Centro-Oeste (3) } \\
\hline & $\mathrm{p} 1$ & p2 & e1 & $\mathrm{e} 2$ \\
\hline p1 & 282,33 & & - & - \\
\hline p2 & 75,18 & 285,46 & - & - \\
\hline e1 & - & - & 199,49 & - \\
\hline $\mathrm{e} 2$ & - & - & - & 186,77 \\
\hline \multicolumn{5}{|c|}{ Sudeste (2) x Centro-Oeste (3) } \\
\hline & p1 & $\mathrm{p} 2$ & e1 & $\mathrm{e} 2$ \\
\hline $\mathrm{p} 1$ & 404,61 & & - & - \\
\hline $\mathrm{p} 2$ & 92,58 & 285,07 & - & - \\
\hline e1 & - & - & 283,68 & - \\
\hline $\mathrm{e} 2$ & - & - & - & 187,87 \\
\hline
\end{tabular}


Tabela 7. Estimativas de herdabilidade direta para as características $1\left(\boldsymbol{h}_{a \mathbf{1}}^{\mathbf{z}}\right)$ e $2\left(\boldsymbol{h}_{a \mathbf{z}}^{\mathbf{z}}\right)$, herdabilidade materna para as características $1\left(\boldsymbol{h}_{m \mathbf{1}}^{\boldsymbol{Z}}\right)$ e $2\left(\boldsymbol{h}_{\mathbf{m} \mathbf{z}}^{\boldsymbol{Z}}\right)$, e correlações genéticas $(R g)$ dos efeitos genéticos diretos dos pesos aos 205 (P205) dias de idade em bovinos Nelore, consideradas como características distintas nas combinações das regiões Norte, Sudeste e Centro-Oeste

\begin{tabular}{cccc}
\hline Característica & Norte/Sudeste & Norte/Centro-Oeste & Sudeste/Centro-Oeste \\
\hline $\boldsymbol{h}_{\boldsymbol{\alpha} \mathbf{1}}^{2}$ & 0,06 & 0,06 & 0,06 \\
$\boldsymbol{h}_{\boldsymbol{\alpha} 2}^{2}$ & 0,06 & 0,12 & 0,11 \\
$\boldsymbol{h}_{\boldsymbol{m} \mathbf{1}}^{2}$ & 0,18 & 0,17 & 0,08 \\
$\boldsymbol{h}_{\boldsymbol{m} \mathbf{2}}^{2}$ & 0,11 & 0,27 & 0,24 \\
$\boldsymbol{R} \boldsymbol{g}$ & $0,85 \pm 0,11$ & $0,96 \pm 0,07$ & $0,95 \pm 0,07$ \\
\hline
\end{tabular}

Tabela 8. Análise conjunta do peso aos 365 (P365) dias de idade, mostrando as herdabilidades diretas para cada região nas diagonais e as correlações genéticas $(\boldsymbol{R g})$ entre a mesma característica considerada como característica distinta entre as regiões Norte (R1), Sudeste (R2) e Centro-Oeste (R3)

\begin{tabular}{ccccccccc}
\hline \multicolumn{1}{l}{ R1xR2 } & \multicolumn{3}{c}{ R1xR3 } & \multicolumn{3}{c}{ R2xR3 } \\
\hline & a1 & a2 & - & a1 & a2 & - & a1 & a2 \\
a1 & $0,28 \pm 0,01$ & - & a1 & $0,29 \pm 0,02$ & - & a1 & $0,30 \pm 0,03$ & - \\
a2 & $0,72 \pm 0,09$ & $0,30 \pm 0,03$ & a2 & $0,83 \pm 0,07$ & $0,34 \pm 0,04$ & a2 & $0,85 \pm 0,08$ & $0,34 \pm 0,03$ \\
\hline
\end{tabular}

Estas divergências devem-se ao número de animais avaliados e aos métodos de manejo os quais os animais foram submetidos, uma vez que os dados são pertencentes a programas de melhoramento genético diferente.

Não foi observado efeito da interação genótipo-ambiente para P205 e P365 quando foram consideradas apenas as regiões Sudeste e Centro-Oeste, que possuem os maiores número de animais dos dados analisados.

A corelação genética entre os animais avaliados nas regiões Norte/Sudeste encontrada para P365 foi baixa, o que indica a presença importante da interação genótipo-ambiente sobre a característica na combinação entre estas regiões.

Desta forma, sugere-se cuidado especial na seleção de animais com maior mérito genético em uma região, pois eles podem não apresentar o mesmo desempenho em outra. Ou seja, as suas progênies podem não apresentar o mesmo desempenho quando criadas em região diferente daquela onde foi realizada a seleção dos reprodutores.

\section{REFERÊNCIAS}

ALENCAR, M.M.; MASCIOLI, A.S.; FREITAS, A.R. Evidências de interação Genótipo x Ambiente sobre características de crescimento em bovinos de corte. Revista Brasileira de Zootecnia, v.34, n.2, p.489-495, 2005.

ARAÚJO, C.V.; BITTENCOURT, T.C.C.; ARAÚJO, S.I.; LÔBO, R.B.; BEZERRA, L.F. Estudo de heterogeneidade de variâncias na 
avaliação genética de bovinos de corte da raça Nelore. Revista Brasileira de Zootecnia, v.40, n.9, p.1902-1908, 2011.

BITTENCOURT, T.C.C.; ROCHA, J.C.M.C.; LÔBO, R.B.; BEZERRA, L.F. Estimação de componentes de (co) variâncas e predição de DEP's para características de crescimento pós desmama de bovinos da raça Nelore, usando diferentes modelos estatísticos.

Arquivo Brasileiro de Medicina Veterinária e Zootecnia, v.54, n.3, p.303-308, 2002.

BOLDMAN, K.G.; KRIESE, L.A.; Van VLECK, L.D. A manual for use of MTDFREML: a set of programs to obtain estimates of variance and covariance. Lincoln: Department of Agriculture, Agricultural Research Service, 1993. 120p.

BOLIGON, A.A.; ALBUQUERQUE, L.G.; RORATO, P.R.N. Associação genética entre pesos e características reprodutivas em rebanhos da raça Nelore. Revista Brasileira de Zootecnia, v.37, n.4, p.596-601, 2008.

CORRÊA, M.B.B.; LAURINO, J.N.; CARDOSO, F.F. Caracterização da interação genótipo ambiente $\mathrm{e}$ comparação entre modelos para ajuste de ganho pós-desmama de bovinos Devon via normas de reação. Revista Brasileira de Zootecnia, v.38, n.8, p.1460-1467, 2009.

ELER, J.P.; FERRAZ, J.B.S.; GOLDEN, B.L.; PEREIRA, E. Influência da interação touro $\mathrm{x}$ rebanho na estimação da correlação entre efeitos genético direto e materno em bovinos da raça Nelore. Revista Brasileira Zootecnia, v.29, p.1642-1648, 2000.
FARIA, C.U.; TERRA, J.P.; YOKOO, M.J.I.; MAGNABOSCO, C.U.; ALBUQUERQUE, L.G.; LÔBO, R.B. Interação genótipo-ambiente na análise genética do peso ao desmame de bovinos Nelore sob enfoque bayesiano. Acta Scientiarum. Animal Sciences, v.33, n.2, p.213-218, 2011.

FRIDRICH, A.B.; SILVA, M.A.; FRIDRICH, D.; CÔRREA, G.S.S.; SILVA, L.O.C.; SAKAGUTI, E.S.; FERREIRA, I.C.; VALENTE, B.D. Interação genótipo $\mathrm{x}$ ambiente $\mathrm{e}$ estimativas de parâmetros genéticos de características ponderais de bovinos Tabapuã. Arquivo Brasileiro de Medicina Veterinária e Zootecnia, v.57, n.5, p.663-672, 2005.

FRIDRICH. A.B.; SILVA, M.A.; VALENTE, B.D.; SOUZA, J.E.R.; CORREAA, G.S.S.; FERREIRA, I.C.; SILVA, L.O.C. Interação genótipo $\mathrm{x}$ ambiente e estimativas de parâmetros genéticos dos pesos aos 205 e 365 dias de idade de bovinos Nelore. Arquivo Brasileiro de Medicina Veterinária e Zootecnia, v.60, n.4, p.917-925, 2008.

INSTITUTO BRASILEIRO DE GEOGRAFIA E ESTATÍSTICA IBGE. Indicadores - Produção Pecuária - Banco SIDRA 2011. Disponível em: < www.ibge.gov.br>. Acesso em: 14 abr. 2012.

LÔBO, R.N.B.; MADALENA, F.E.; VIEIRA, AR. Average estimates of genetic parameters for beef and dairy cattle in tropical regions. Animal Breeding Abstracts, v.68, n.6, p.433461, 2000.

LOPES, J.S.; RORATO, P.R.N.; WEBER, T.; BOLIGON, A.A.; COMIN, J.G.; DORNELLES, M.A. Efeito da Interação Genótipo-Ambiente sobre o peso ao nascimento, aos $205 \mathrm{e}$ 
aos 550 dias de idade de bovinos da raça Nelore na Região Sul do Brasil.

Revista Brasileira de Zootecnia, v.37, n.1, p.54-60, 2008.

RIBEIRO, S.; BALIEIRO, J.B.S.; FERRAZ, V.B.; PEDROSA, V.B.; MATTOS, E.C. Influência da interação genótipo $\mathrm{x}$ ambiente sobre o peso à desmama em bovinos da raça Nelore.

Arquivo Brasileiro de Medicina Veterinária e Zootecnia, Pirassununga, v.61, n.3, p.668-675, 2009.

SARMENTO, J.L.R.; PIMENTA FILHO, E.C.; RIBEIRO, M.N.; MARTINS FILHO, R. Efeitos Ambientais e Genéticos sobre o Ganho em Peso Diário de Bovinos Nelore no Estado da Paraíba. Revista Brasileira de Zootecnia, v.32, n.2, p.325-330, 2003.

SOUZA, J.C.; DOSKA, M.C.; SILVA. L.O.C.; GONDO, A.; RAMOS, A.A.; MALHADO, C.H.M.; SANTOS, I.W.; FREITAS, J.A.; FERRAZ FILHO, P.B.; SERENO, J.R.B. Interacción genótipo $\mathrm{x}$ ambiente sobre el peso al destete de bovinos Nelore em Brasil. Archivos de Zootecnia, v.57, n.218, p.171-177, 2008.

SAS Institute. Statistical analysis system. Versão 6.12 para windows. Cary, 1997. 1290p.
TEIXEIRA, R.A.; ALBUQUERQUE, L.G.; ALENCAR, M.M.; DIAS, L.T. Interação genótipo-ambiente em cruzamentos de bovinos de corte.

Revista Brasileira de Zootecnia, v.35, n.4, p.1677-1683, 2006.

TORAL, F.L.B.; SILVA, L.O.C.; MARTINS, E.N.; GONDO, A.; SIMONELLI, S.M. Interação genótipo $\mathrm{x}$ ambiente em características de crescimento de bovinos da raça Nelore no Mato Grosso do Sul. Revista

Brasileira de Zootecnia, v.33, n.6, p.1445-1455, 2004.

YOKOO, M.J.I.; ALBUQUERQUE, L.G.; LÔBO, R.B.; SAINZ, R.D.;

CARNEIRO JÚNIOR, J.M.;

BEZERRA, L.A.F.; ARAÚJO, F.R.C. Estimativas de parâmetros genéticos para altura do posterior, peso e circunferência escrotal em bovinos da raça Nelore. Revista Brasileira de Zootecnia, v.36, n.6, p.1761-1768, 2007.

Data de recebimento: $31 / 08 / 2012$

Data de aprovação: 06/12/2012 